Original Article (short paper)

\title{
Heart rate responses of a master athlete during ultra-endurance swimming
}

\author{
Renata Rodrigues Teixeira de Castro ${ }^{1}$ \\ ${ }^{1}$ Universidade Iguaçu, Escola de Medicina, Nova Iguaçu, RJ, Brasil.
}

\begin{abstract}
Aim: Swimming races outside swimming pools take place all over the world, but studies focusing on their physiological aspects are scarce. In fact, rules forbid any direct contact with swimmers during sanctioned events. This case report presents heart rate responses of a master athlete during an ultra-endurance open water solo swim and analyzes results based on a prior cardiopulmonary exercise test. Methods: The swimmer performed a cardiopulmonary exercise test on a treadmill, following a ramp protocol, with continuous electrocardiogram recording. The athlete performed a $36 \mathrm{~km}$ swimming race with continuous heart rate recording. Description of swimming heart rate accordingly to heart rate training zones and correlation analysis between HR and swim speed was assessed. Results: The athlete swam the $36 \mathrm{~km}$ event in 11 hours, 16 minutes and 15 seconds. Most of the swim time was spent in training zone 3 $(55 \%)$, and only $2 \%$ of race time was spent in zone 5 . Swimming speed $(3.2 \pm 0.7 \mathrm{~km} / \mathrm{h})$ failed to correlate with HR during the event $\left(r^{2}=0.1334, p>0.05\right)$. Conclusion: Although HR did not correlate with swimming speed it successfully indicated exercise intensity during this $36 \mathrm{~km}$ event. These results can be used in the preparation and monitoring of other solo long-distance swimmers.
\end{abstract}

Keywords: open water, swimming, heart rate, physiology, master.

\section{Introduction}

Swimming events taking place in rivers, lakes, oceans, channels, seas, bays or alike places are considered open water swimming (OWS) events. The only OWS Olympic event is the $10 \mathrm{~km}$ race, but the Fe'de'ration Internationale de Natation (FINA) officially promotes $5 \mathrm{~km}$ and $25 \mathrm{~km}$ of OWS events in its championships.

Other non-official OWS races take place all over the world ${ }^{1}$. Nevertheless, studies focusing on OWS, especially ultra-endurance swimming, are rare $^{2}$, and most of them focused on hypothermia ${ }^{1,3,4}$. Information about physiological responses during ultra-endurance swimming events is scarce ${ }^{2,5}$, as rules forbid any direct contact with swimmers during sanctioned events.

Over the few published case studies ${ }^{2-5}$ describing open water swimmer's physiological parameters during races, only one focused on heart rate (HR) response. Judelson, Bagley, Schumacher, Wiersma ${ }^{5}$ described that a 24-years-old female swimmer kept a constant HR (148155 beats/min) during the $32,2 \mathrm{~km}$ Catalina Channel swim despite fluctuation in perceptual stressors.

When studying HR responses to exercise, special attention must be paid to the athlete's age, as maximal HR tends to decline over the years. Although long-distance swimmers competing from $26 \mathrm{~km}$ to $35 \mathrm{~km}$ usually get to peak performance in the $4^{\text {th }}$ decade of life ${ }^{6}$, FINA considers swimmers who are older than 25 years-old as master athletes.
This case report presents HR responses of a master athlete with hypertension during an ultra-endurance open water solo swim and analyzes the results in the light of a cardiopulmonary exercise test.

\section{Case Report}

\subsection{Participant}

A 39-years-old male swimmer with three-year experience in OWS (weight: $83 \mathrm{~kg}$; height: 1,70 m; body mass index: $28,72 \mathrm{~kg} / \mathrm{m}^{2}$ ) participated in the "do Leme ao Pontal Swim" in Rio de Janeiro's coast. He had hypertension for several years and was on losartan therapy $(50 \mathrm{mg} /$ day). Blood pressure levels have been well controlled in the last five years, and there was no end-organ damage.

Ethics

The investigation conforms with the principles outlined in the Declaration of Helsinki. The study was approved by the IRB of Universidade Iguaçu (CAAE 14088119.0.0000.8044). The patient signed informed consent before enrolment.

\subsection{Cardiopulmonary exercise testing}

Three months before the event, the patient underwent a maximal cardiopulmonary exercise test (Handymet, MDI, Brazil) following an incremental ramp protocol on a treadmill (ATL, Inbrasport, Brasil). The athlete star- 
ted the exercise test at $6 \mathrm{~km} / \mathrm{h}$ and $1 \%$ grade and reached maximal effort at $14,1 \mathrm{~km} / \mathrm{h}$ and $1 \%$ grade. Minute ventilation (Ve), oxygen consumption (VO2) and carbon dioxide production $(\mathrm{CO} 2)$ were registered every $10 \mathrm{~s}$. Derived variables were calculated online (Handymet studio software, MDI, Brazil).

The ventilatory threshold was identified by the combination of the following methods ${ }^{7}$ : at the point of the first upward inflection of the ventilation vs. time curve; at the beginning of a consistent increase in the ventilatory equivalent for $\mathrm{O} 2$ (minute ventilation/oxygen consumption) without a concomitant increase in the ventilatory equivalent for carbon dioxide (minute ventilation/carbon dioxide production); and at the beginning of an increase in expired oxygen fraction. The ventilatory threshold was considered as the point identified by at least two of these three criteria.

Respiratory compensation point was identified at the point of the second upward inflection of the ventilation vs. time curve, which was concomitant to the beginning of a consistent increase in the ventilatory equivalent for carbon dioxide (minute ventilation/carbon dioxide production). The maximum value of each variable during the final $30 \mathrm{~s}$ of the exercise was used as peak variables.

During the cardiopulmonary exercise test, 12-lead electrocardiogram was continuously recorded (Xcribe, Mortara, USA) and HR registered. HR limits for each training zone were calculated as percentages of anaerobic threshold's HR (158 bpm), as follows : zone $1(\leq 68 \%$ : $\leq$ 107 beats/min ); zone 2 (69\% to $83 \%$ : $108-131 \mathrm{bpm}$ ); zone 3 (84\% to $94 \%$ : $132-149 \mathrm{bpm})$; zone 4 (95\% to $105 \%$ : 150-166 bpm); zone 5 (> 105\%: > $167 \mathrm{bpm}$ ). Test results are shown in Table 1.

\subsection{HR monitoring}

We intended to monitor HR throughout the role swim with a Polar M Vantage watch (Polar Electro, Finland) positioned in the patient's left arm. Unfortunately, the watch's power went out in the last hour of the swim (Figure 1). Nevertheless, it recorded global positioning system data, HR and swim pace each second for $10 \mathrm{~h}$, $16 \mathrm{~min}$. and $35 \mathrm{~s}$, leaving only the last hour unrecorded.

\subsection{Do Leme ao Pontal swimming event}

The athlete swam $36 \mathrm{~km}$ from Leme to Pontal (Figure 1), in Rio de Janeiro's coast, wearing only a swimming suit, cap, and goggles. There was no use of insulating or buoyant material, nor any unnatural assistance to the swimmer. He started at 11:04 PM with a water temperature of $23{ }^{\circ} \mathrm{C}$. At 30 -min. intervals, the athlete rested (treading water) for less than one minute, drinking water or consuming bread, fruits, smoothies or solutions with caffeine, carbohydrate and electrolytes.

Statistical analysis

Continuous variables are reported as means and standard deviations. A simple correlation analysis between HR and swim speed was assessed. A two-sided Pvalue $<0.05$ was considered significant.

All statistical analyses were performed with STATA 14.2 (StataCorp, Texas, USA).

\section{Results}

The athlete swam the $36 \mathrm{~km}$ "Do Leme ao Pontal swimming event" in $11 \mathrm{~h}, 16 \mathrm{~min}$. and $15 \mathrm{~s}$. Monitoring was available in the first $32,629 \mathrm{~m}$ of the swim, with an average of 62 strokes/minute and an average pace of $1 ' 53 \% / 100 \mathrm{~m}$. Swimming speed $(3.2 \pm 0.7 \mathrm{~km} / \mathrm{h})$ failed to correlate with HR during the event $\left(r^{2}=0.1334, p>0.05\right.$; Figure 2).

There were 36993 recorded HR values (one value/ second). Most of them were below the athlete's anaerobic threshold ( $n=34420,93 \%$ of the time; Figure 3 ). The athlete has started the race with an HR of 98 beats $/ \mathrm{min}$ and the mean recorded HR was $133 \pm 19$ beats/minute. Maximal recorded HR was 204 beats/minute. Most of the swim time was spent in training zone $3(55 \%)$, and only $2 \%$ of race time was spent in zone 5 (Figure 2). The higher HRs recordings occurred from $5: 45^{\prime}$ to $6: 48^{\prime}$ and can be explained by the rough sea currents at this part of the race.

\section{Discussion}

The athlete became the 25th solo swimmer to successfully complete this event. To our knowledge, this is the first report of a patient with hypertension completing an ultra-endurance swim. Most cardiology guidelines agree that, in the absence of target-organ damage, stage-1 hypertension should not prevent athlete participation in competitive sport ${ }^{9}$.

Previous reports used age-based equations to analyze exercise intensity ${ }^{5,6}$. Although practical, these formulas

Table 1 - Cardiopulmonary exercise test values from the participant, three months before the open water swim.

\begin{tabular}{lccc}
\hline Parameter & Anaerobic threshold & Respiratory compensation point & Peak exercise \\
\hline Heart rate (beats $/ \mathrm{min})$ & 158 & 176 & 190 \\
Oxygen uptake $(\mathrm{mL} / \mathrm{kg} / \mathrm{min})$ & 27.7 & 34.1 & 37.4 \\
Oxygen uptake $(\%)$ & 74 & 91 & 100 \\
\hline
\end{tabular}




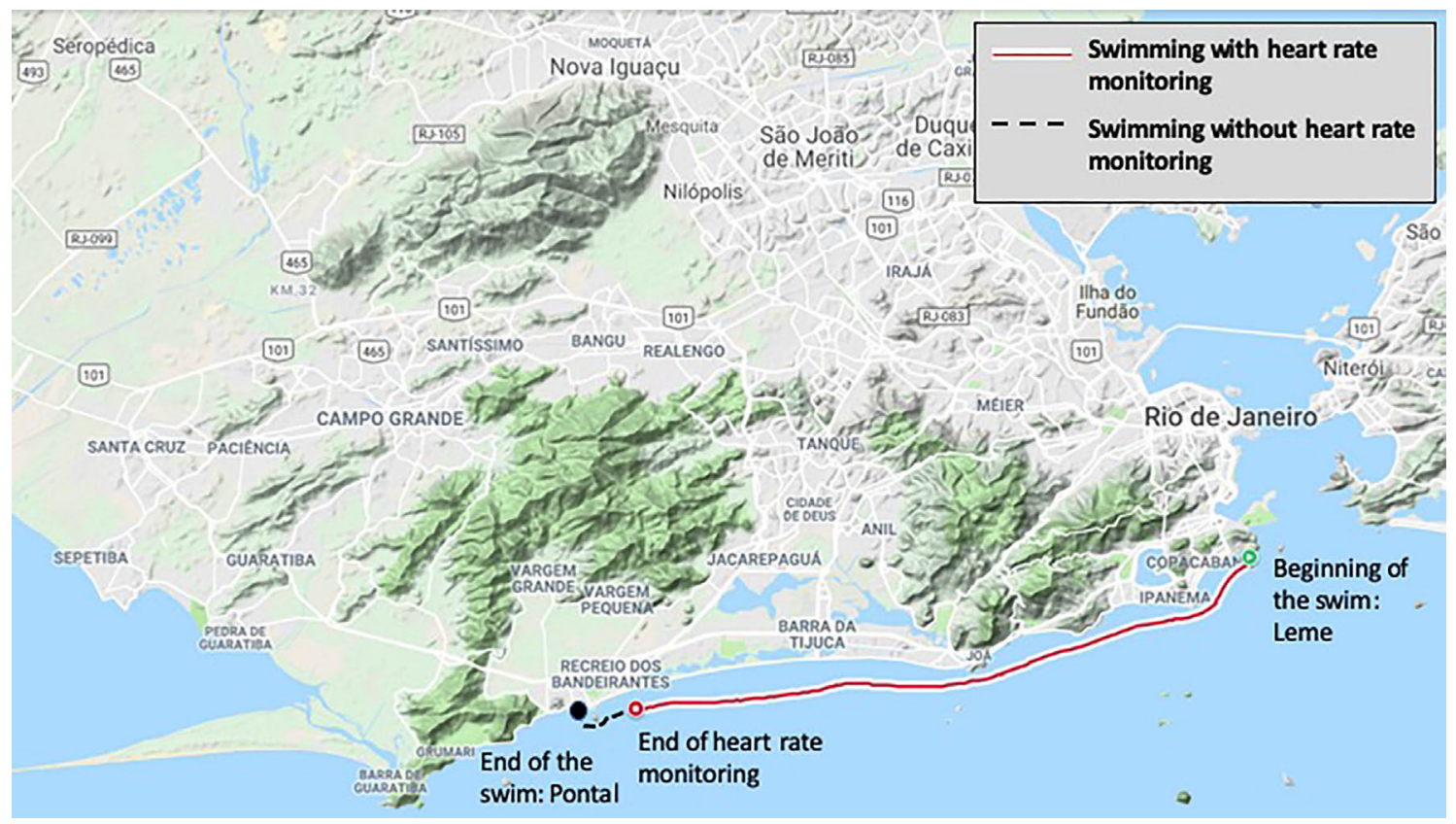

Figure 1 - Rio de Janeiro's coastal map showing global position system tracking of the monitored period.

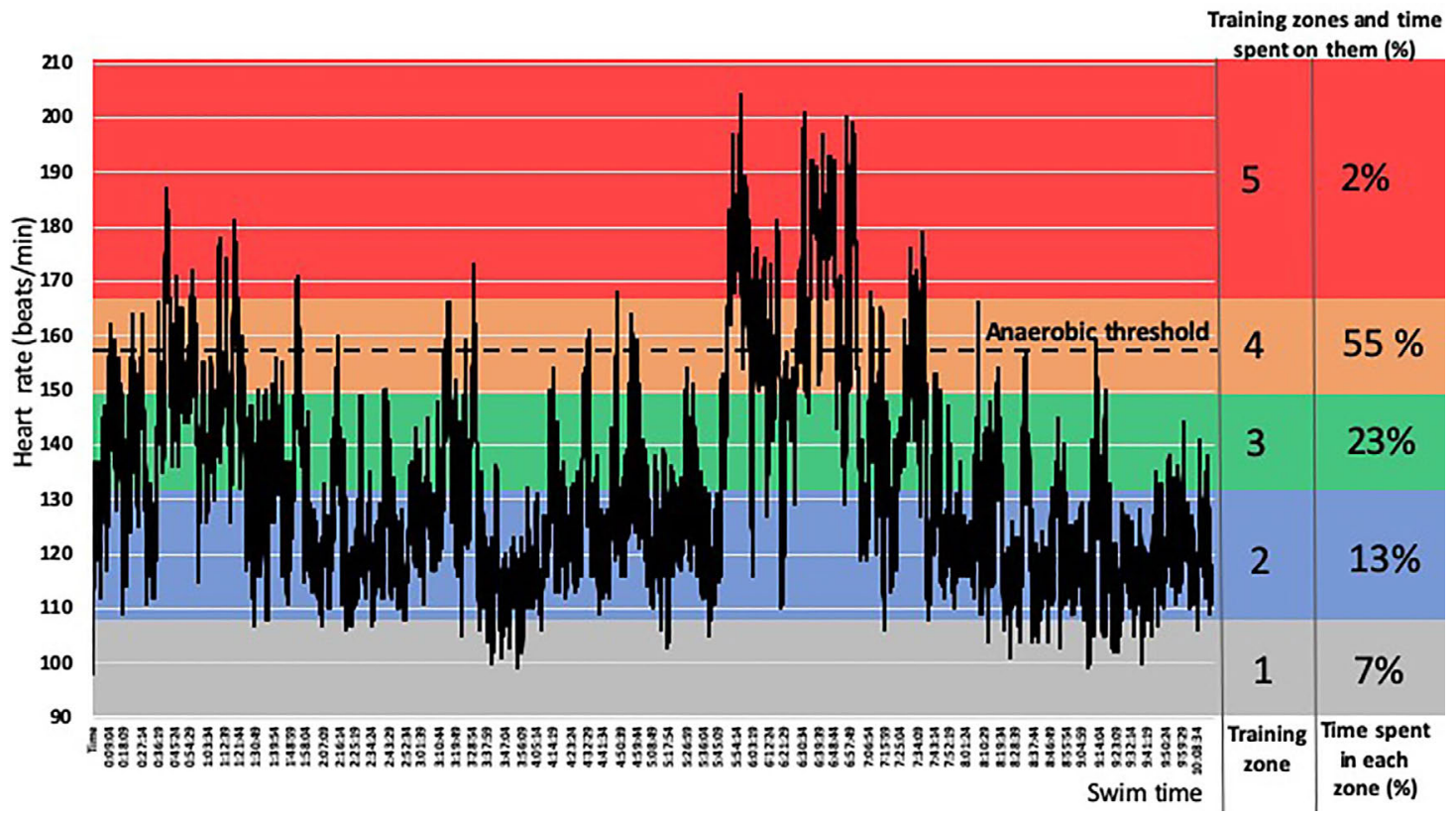

Figure 2 - Heart rate and swimming speed of the athlete during the event

may present errors and don't show the exact condition of the athlete. In the present study, we analyzed exercise intensity by comparison of recorded HR during the race and the HR from the cardiopulmonary exercise test, showing that aerobic metabolism was predominant during the event.

It is important to note that HR responses may differ accordingly to exercise modality. We have previously found no differences in maximal heart rate during progressive cardiopulmonary exercise tests performed on cycle or arm ergometers ${ }^{10}$. Hauber, Sharp, Franke ${ }^{11}$ assessed swimmers' HR responses to submaximal and maximal treadmill running and free swimming and found that despite peak heart rate and oxygen uptake are mode-specific, exercising at a given submaximal oxygen uptake elicits similar HR regardless of the mode of exercise. Thus, the study by Hauber, Sharp, Franke ${ }^{11}$ validates the use of land-based determined target HR zones while swim$\operatorname{ming}^{11}$, as done in the present study. 


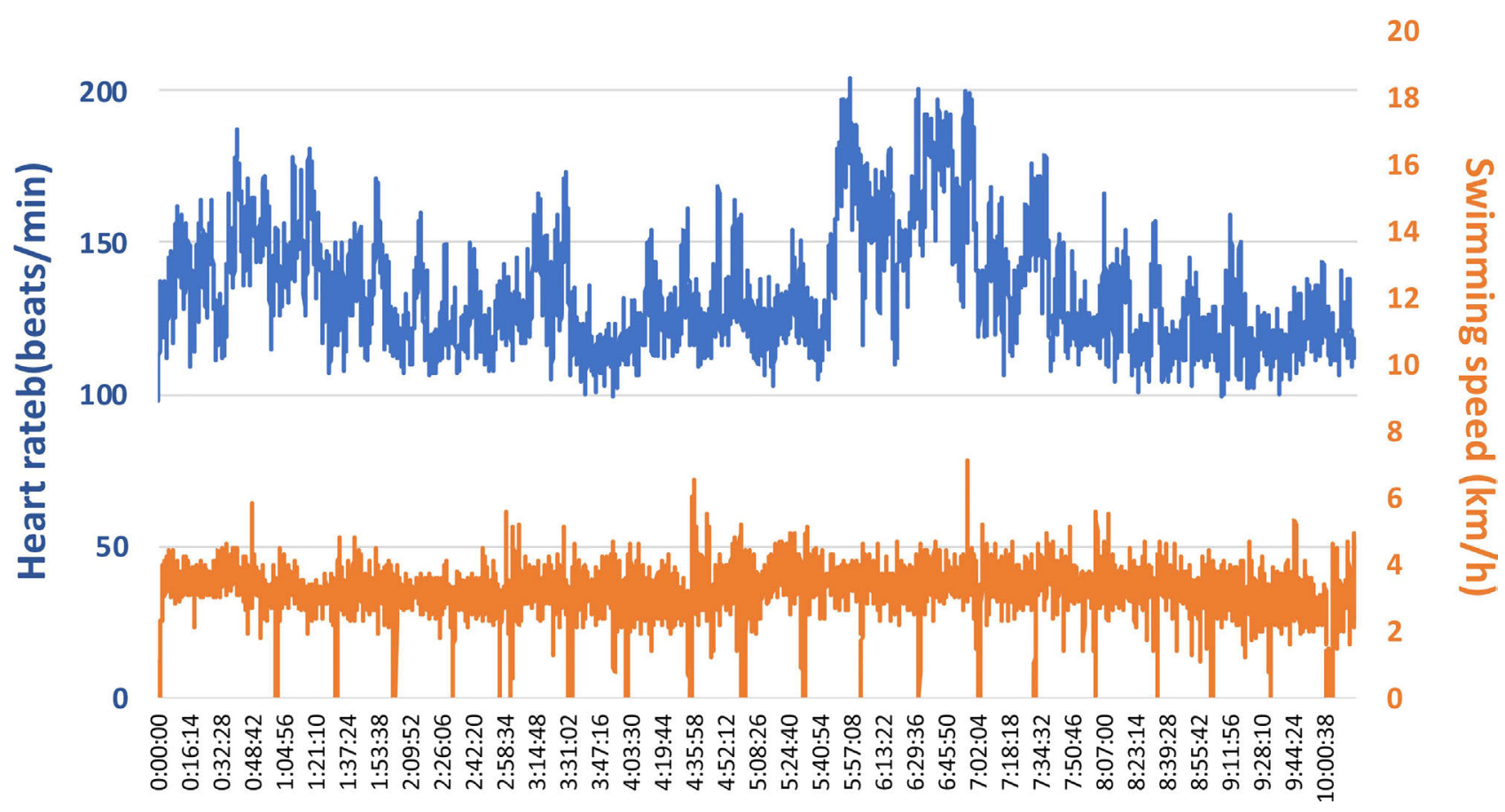

Figure 3 - Athlete's heart rate and heart rate training zones during the event.

Swimming speed was obtained from the variation in global position through time and could not account for ocean velocity and direction, which definitely influence swimming speed. Thus, depending on ocean currents, different levels of effort are needed to keep the same swimming speed $^{5}$ and this explains the lack of correlation between HR and swimming speed in our study.

\section{Conclusion}

This is the first report to continuously record HR of a master athlete with hypertension during an ultra-endurance swimming event. Although HR did not correlate with swimming speed it successfully indicated exercise intensity during this $36 \mathrm{~km}$ event. Most of the swimming was performed below the anaerobic threshold, indicating a predominantly aerobic event. These results can be used in the preparation and monitoring of other solo long-distance swimmers.

\section{References}

1. Eichenberger E, Knechtle B, Knechtle P, Rüst CA, Rosemann T, Lepers R. Best performances by men and women open-water swimmers during the 'English Channel Swim' from 1900 to 2010. J Sports Sci. 2012;30:1295-301.

2. Baldassarre R, Bonifazi M, Zamparo P, Piacentini MF. Characteristics and Challenges of Open-Water Swimming Performance: A Review. Int J Sports Physiol Perform. 2017;12:1275-84.

3. de Castro RR, da Nobrega AC. Hypothermia in open-water swimming events: a medical risk that deserves more atten- tion. Wilderness Environ Med. 2009;20:394-5; author reply 5 .

4. Castro RR, Mendes FS, Nobrega AC. Risk of hypothermia in a new Olympic event: the $10-\mathrm{km}$ marathon swim. Clinics (Sao Paulo). 2009;64:351-6.

5. Judelson DA, Bagley JR, Schumacher JM, Wiersma LD. Cardiovascular and Perceptual Responses to an Ultraendurance Channel Swim: A Case Study. Wilderness Environ Med. 2015;26:359-65.

6. Zingg MA, Rüst CA, Rosemann T, Lepers R, Knechtle B. Analysis of swimming performance in FINA World Cup long-distance open water races. Extrem Physiol Med. 2014;3:2.

7. Ross RM. ATS/ACCP statement on cardiopulmonary exercise testing. Am J Respir Crit Care Med. 2003;167:1451; author reply

8. Maynard T. Exercise: Part II. Translating the exercise prescription. Diabetes Educ. 1991;17:384-95.

9. Black HR, Sica D, Ferdinand K, White WB. American Heart Association Electrocardiography and Arrhythmias Committee of Council on Clinical Cardiology CoCDiY, Council on Cardiovascular and Stroke Nursing, C.uncil on Functional Genomics and Translational Biology, and American College of Cardiology. Eligibility and Disqualification Recommendations for Competitive Athletes With Cardiovascular Abnormalities: Task Force 6: Hypertension: A Scientific Statement from the American Heart Association and the American College of Cardiology. Circulation. 2015;132: e298-302.

10. Castro RR, Pedrosa S, Nobrega AC. Different ventilatory responses to progressive maximal exercise test performed with either the arms or legs. Clinics (Sao Paulo). 2011;66:1137-42. 
11. Hauber C, Sharp RL, Franke WD. Heart rate response to submaximal and maximal workloads during running and swimming. Int J Sports Med. 1997;18:347-53.

\section{Corresponding author}

Renata Castro Universidade Iguaçu, School of Medicine, Av Abilio Augusto Tavora, Nova Iguacu, Brazil. E-mail: castrorrt@gmail.com.
Manuscript received on December 2, 2019

Manuscript accepted on February 14, 2020

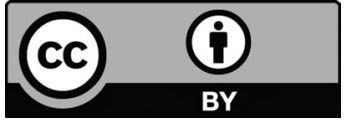

Motriz. The Journal of Physical Education. UNESP. Rio Claro, SP, Brazil - eISSN: 1980-6574 - under a license Creative Commons - Version 4.0 